\title{
Positioning of the Fermi Level in Graphene Devices with Asymmetric Metal Electrodes
}

\author{
Bum-Kyu Kim, ${ }^{1}$ Eun-Kyoung Jeon, ${ }^{1}$ Ju-Jin Kim, ${ }^{1}$ and Jeong-O Lee ${ }^{2}$ \\ ${ }^{1}$ Department of Physics, Institute of Physics and Chemistry, Chonbuk National University, Jeonju 561-756, Republic of Korea \\ ${ }^{2}$ Fusion-Biotechnology Research Center, Korea Research Institute of Chemical Technology, Daejeon 305-600, Republic of Korea
}

Correspondence should be addressed to Ju-Jin Kim, jujinkim@chonbuk.ac.kr

Received 15 June 2010; Accepted 7 July 2010

Academic Editor: Rakesh Joshi

Copyright ( 2010 Bum-Kyu Kim et al. This is an open access article distributed under the Creative Commons Attribution License, which permits unrestricted use, distribution, and reproduction in any medium, provided the original work is properly cited.

To elucidate the effect of the work function on the position of the Dirac point, we fabricated graphene devices with asymmetric metal contacts. By measuring the peak position of the resistance for each pair of metal electrodes, we obtained the voltage of the Dirac point $V_{g}^{\text {Dirac }}(\mathrm{V})$ from the gate response. We found that the position of $V_{g}^{\text {Dirac }}(\mathrm{V})$ in the hybrid devices was significantly influenced by the type of metal electrode. The measured shifts in $V_{g}^{\text {Dirac }}(V)$ were closely related to the modified work functions of the metal-graphene complexes. Within a certain bias range, the Fermi level of one of the contacts aligned with the electron band and that of the other contact aligned with the hole band.

\section{Introduction}

The electrical properties of nanosized devices are significantly influenced by the characteristics of the electrical contacts in the devices. In devices in which a carbon nanotube (CNT) conducting channel is in contact with a metal, the carrier type of the device (hole or electron) can be selected by appropriate positioning of the Fermi level at the interface between the metal and the CNT channel [1-4]. The Schottky barrier at the metal electrode/semiconducting channel interface is determined by the energy difference between the metal work function and the electron affinity of the channel. Metal electrodes with low work functions are typically used for electron injection into the conduction band whereas metal electrodes with high work functions are adequate for hole injection into the valence band. In practice, however, it is difficult to find appropriate electrode metals with optimal work functions that are suitable for use with particular conducting channels $[5,6]$. If the work function of the metal electrode could be varied systematically, it would be possible to precisely tailor the electrical properties of a device, thereby modifying the energy level alignment near the electrode/semiconductor interface.

On the other hand, substitutional impurity doping techniques used in the conventional bulk semiconductor industry encounter many challenges when applied to carbonbased nanodevices, such as CNTs and graphene devices, due to the nonuniformity of the doping and the perfect covalent bonding structure of the carbon nanostructures. Precise control over the electrical properties of CNT devices is essential for future electronic device development [7]. As an alternative to impurity doping, contact doping may be used to modify the barrier height between carbon-based conducting channels and metal electrodes. Charge transport in most CNT field-effect transistors (FETs) is strongly influenced by the Schottky barrier between the nanotubes and the contact electrode. Under normal conditions, CNTFETs fabricated to date have shown only $p$-type operation [8]. The reason why CNT-FETs exhibit characteristic $p$ type behavior has long been a topic of debate $[9,10]$. Previously, hole doping by environmental oxygen molecules was believed to be the principal cause of the $p$-type behavior of CNT-FETs $[11,12]$. However, it is now generally agreed that $p$-type behavior arises from the low-lying Schottky barrier for hole transport at a high work function metal-CNT interface [13]. The position of the Fermi level at the interface between the metal electrode and the CNT channel should be a key parameter governing the transport characteristics in these devices. 


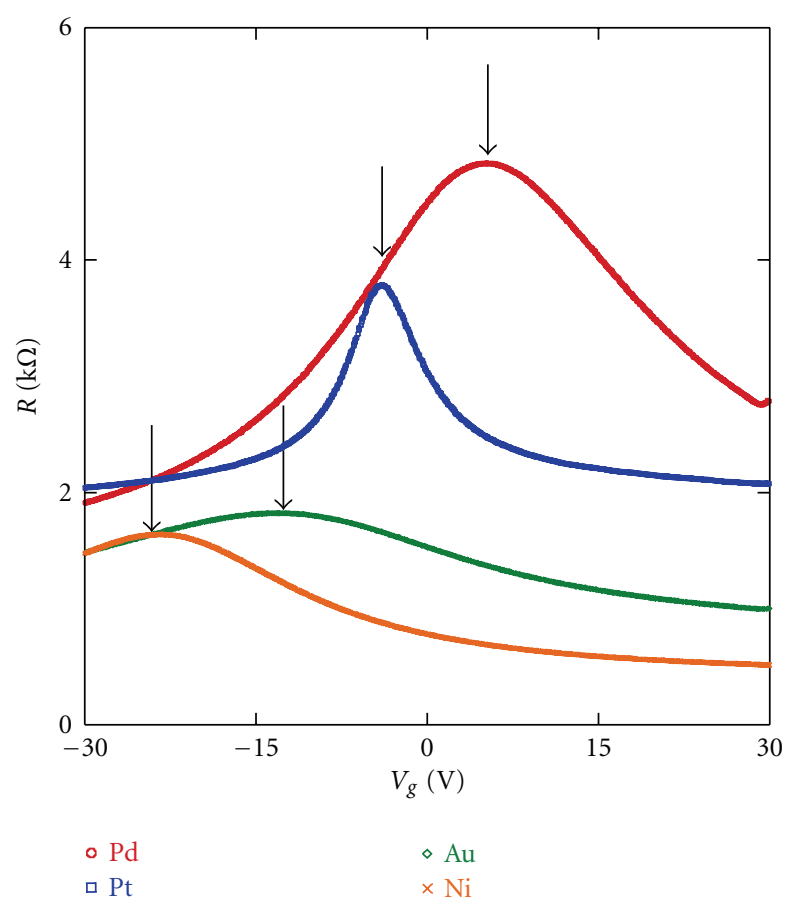

FIgURE 1: Two-probe electronic transfer characteristics measured in graphene transistors with various metal contacts. The arrows indicate the positions of the Dirac point voltages, $V_{g}^{\text {Dirac }}(\mathrm{V})$. The specific values of $V_{g}^{\text {Dirac }}(\mathrm{V})$ were $+1.26 \mathrm{~V}(\mathrm{Pd}),-3.92 \mathrm{~V}(\mathrm{Pt})$, $-13.12 \mathrm{~V}(\mathrm{Au})$, and $-24.33 \mathrm{~V}(\mathrm{Ni})$.

Because graphene is a zero-gap semiconductor with a linear dispersion relation near the Fermi level [14], graphene devices with metal electrodes may show Fermi level positioning effects similar to those observed in CNT devices. By adjusting the Fermi level pinning position, we can intentionally select whether the charge carrier passing through the contact barrier is an electron or hole. The controlled adjustment of the Dirac point is not only of scientific interest but also is employed in the fabrication of graphenebased devices. Previous studies have modulated the Dirac point by varying the concentration of external dopants or by applying an electrostatic bias $[15,16]$. However, this type of Dirac point adjustment arises from channel doping, not from Fermi level positioning effects.

In previous transport experiments, the position of the Dirac point has appeared to be arbitrary. The effects of metal contact, edges, defects, and adsorbed species were thought to be intertwined in some nonlinear fashion and, therefore, were difficult to separate [17-21]. The effects of the metal contacts were particularly intriguing because most transport measurements are expected to depend fundamentally on the metal-graphene interface $[22,23]$. In the present paper, we investigated the role of the metal work function on the position of the Dirac charge neutrality point $V_{g}^{\text {Dirac }}(\mathrm{V})$ by measuring the gate transfer characteristics of graphene transistors with asymmetric metal contacts. A variety of metal electrodes that bind physically or chemically to graphene were employed as contact electrodes, and we confirmed that

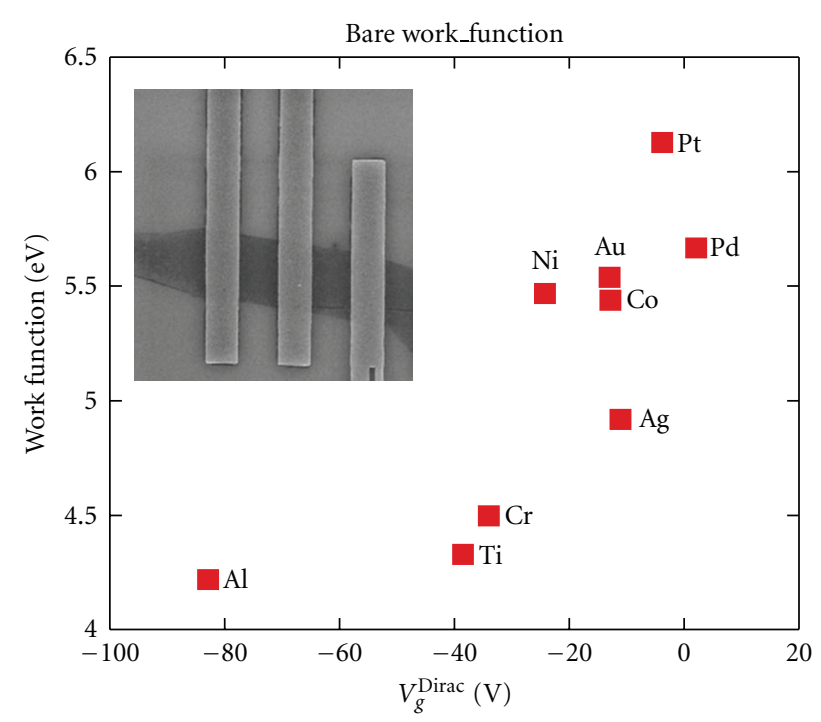

Figure 2: Averaged voltages of Dirac points in the gate response as a function of the metal work function. Inset: scanning electron micrograph image of a typical graphene field effect transistor used in this experiment.

the Dirac point $V_{g}^{\text {Dirac }}(\mathrm{V})$ could be modified by varying the metal work function and the metal-graphene interaction. By employing asymmetric metal electrodes, we demonstrated that it was possible to simultaneously inject different types of charge carrier into the same graphene channel.

\section{Experimental}

Graphene sheets were micromechanically cleaved from a highly oriented pyrolytic graphite (HOPG) sample. Graphene devices were prepared on a heavily doped Si substrate with a $300 \mathrm{~nm}$ thick, thermally grown $\mathrm{SiO}_{2}$ layer. Once a suitable graphene sheet was characterized by optical microscopy and atomic force microscopy, deep UV lithography and electron-beam (e-beam) lithography were used to generate electrode patterns on the selected graphene sheet. To minimize chemical contamination, we used only a PMMA resist during the fabrication process. Source and drain electrodes were formed by depositing one of a variety of metals with different work functions by either sputtering (for $\mathrm{Co}, \mathrm{Ni}, \mathrm{Ti}, \mathrm{Cr}, \mathrm{Pd}$, or $\mathrm{Au}$ ) or electron-beam evaporation techniques (for $\mathrm{Pt}, \mathrm{Ag}, \mathrm{Au}, \mathrm{Ti}, \mathrm{Pd}$, or $\mathrm{Al}$ ). The film deposition technique did not significantly affect the position of the Dirac point. Devices with hetero-metallic contacts were subjected to two identical e-beam lithography procedures. To discriminate the effect of metal work function, all fabrication parameters were kept constant as much as possible, and the same fabrication process and device layout were used. The inset of Figure 2 shows a scanning electron microscopy image of a typical graphene transistor with metal electrodes. A heavily doped Si substrate was used as a back gate to control the carrier concentration in the graphene devices, and the channel lengths of the devices were kept at approximately $2 \mu \mathrm{m}$. 


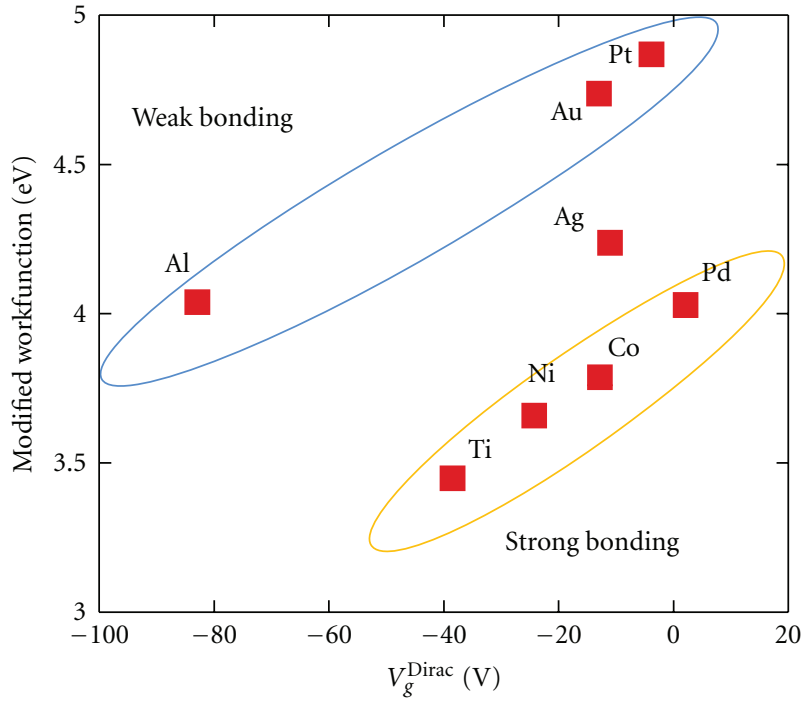

Figure 3: Position of the Dirac point in the gate response in relation to the modified work function of a metal-graphene complex. Two classes of metal-those that physisorb and those that chemisorb onto graphene-are clearly distinguished, with the exception of Ag.

\section{Results and Discussion}

First, we address the transport characteristics of the graphene devices with symmetric source and drain contact electrodes. The channel doping effects due to the adsorption of molecules on the surfaces of the graphene channel were minimized by preparing all devices under identical fabrication conditions, with the only difference being the identity of the metal electrode. We measured the gate-dependent resistance of the graphene devices, as shown in Figure 1. The positions of the resistance peaks designated by arrows correspond to the Dirac points at the metal-graphene interfaces, and these positions depended on the type of metal electrode. Only the Pd-contact device showed a positive peak voltage, implying that the Fermi level was aligned with the hole band of the graphene channel. In contrast, other materials $(\mathrm{Pt}, \mathrm{Au}$, and $\mathrm{Ni}$ ) showed a negative Dirac peak voltage.

The relation between the peak position and the metal electrode was inferred by plotting the average voltage of the Dirac point $V_{g}^{\text {Dirac }}(\mathrm{V})$ of the gate response as a function of the bare metal work function, as shown in Figure 2. $V_{g}^{\text {Dirac }}$ $(\mathrm{V})$ was obtained from the average value measured in 4-6 devices. Because the metal contact, as an electron reservoir, determines the Fermi level of the graphene, $V_{g}^{\text {Dirac }}(\mathrm{V})$ may be related to the metal work function. At first glance, $V_{g}^{\text {Dirac }}$ (V) appears to be closely correlated with the magnitude of the bare metal work function [24]. However, some deviations from the expected values are readily identified, especially for contact materials with good wetting properties, such as $\mathrm{Ti}$, $\mathrm{Cr}$, and Co. Because the density of states in graphene is much lower than that in an electrode metal, even small amounts of electron transfer near the interface will significantly shift the Fermi level [22]. Thus, the interface charge transfer between the metal and graphene is another governing parameter

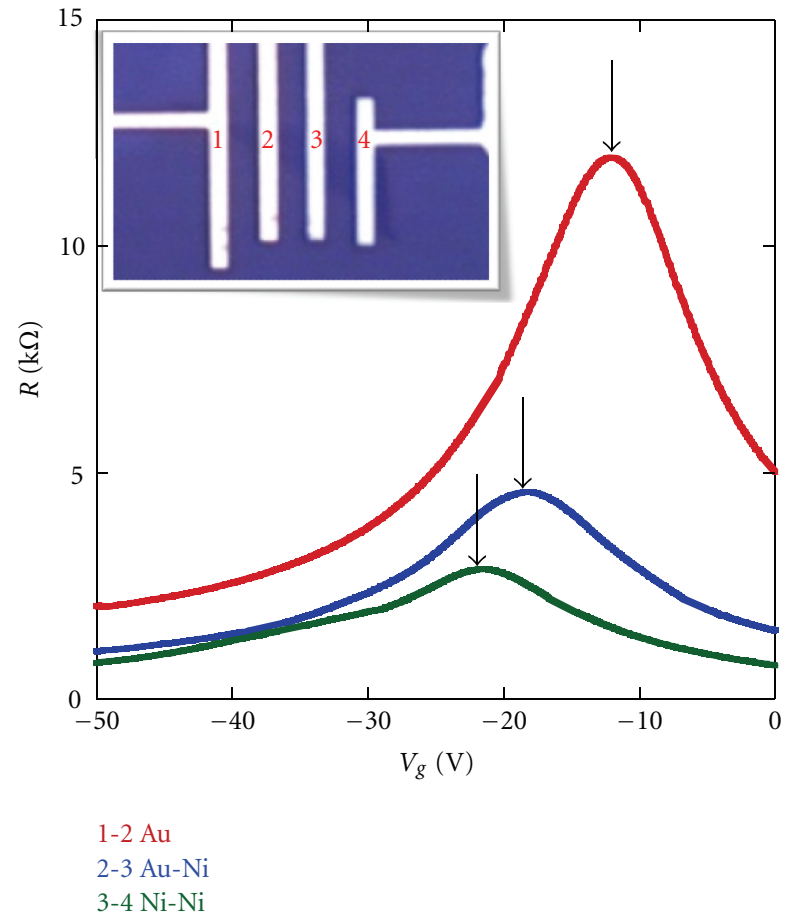

FIGURE 4: Gate response curves measured from a symmetric $\mathrm{Au}-$ contact graphene device (1-2), a Ni-contact device (3-4), and an asymmetric-contact device containing $\mathrm{Ni}$ and $\mathrm{Au}$ contacts (2-3). Inset: Optical microscope image of graphene with asymmetric hetero-metal electrodes $(\mathrm{Au}, \mathrm{Ni})$.

for the Fermi level alignment, particularly in regards to the good wetting properties of the metal electrode to the graphene channel. Accordingly, we classified the metals into two groups: those physisorbed onto graphene $(\mathrm{Al}, \mathrm{Au}$, and $\mathrm{Pt}$ ) and those chemisorbed onto graphene ( $\mathrm{Ti}, \mathrm{Ni}, \mathrm{Co}$, and Pd). In previous theoretical studies [22, 23], the electronic structure of the metal surface in contact with the graphene over layer was investigated, and the movement of the Fermi level of graphene was obtained with respect to the work function of the metal-graphene complex.

We replotted the average $V_{g}^{\text {Dirac }}(\mathrm{V})$ of the gate response as a function of the "modified work function" of the metalgraphene complex, as shown in Figure 3. The modified work function values in Figure 3 were derived from $[22,23]$. When plotted in this way, $V_{g}^{\text {Dirac }}(\mathrm{V})$ could be separated into two groups-physisorbed (weak bonding) metal electrodes and chemisorbed (strong bonding) metal electrodes-with the exception of Ag. Within each of these groups, the movement of the Dirac point of the gate response was almost directly proportional to the work function of the metal-graphene complex. The Ag-contact device, which did not fall into either group, appeared to show a much stronger binding behavior than what would be predicted by theoretical calculations [22]. The deviation of the Ag electrode device from the expected behavior may originate from some other type of strong bonding near the interface. Further theoretical and experimental studies are required to understand the discrepancy of this system. 


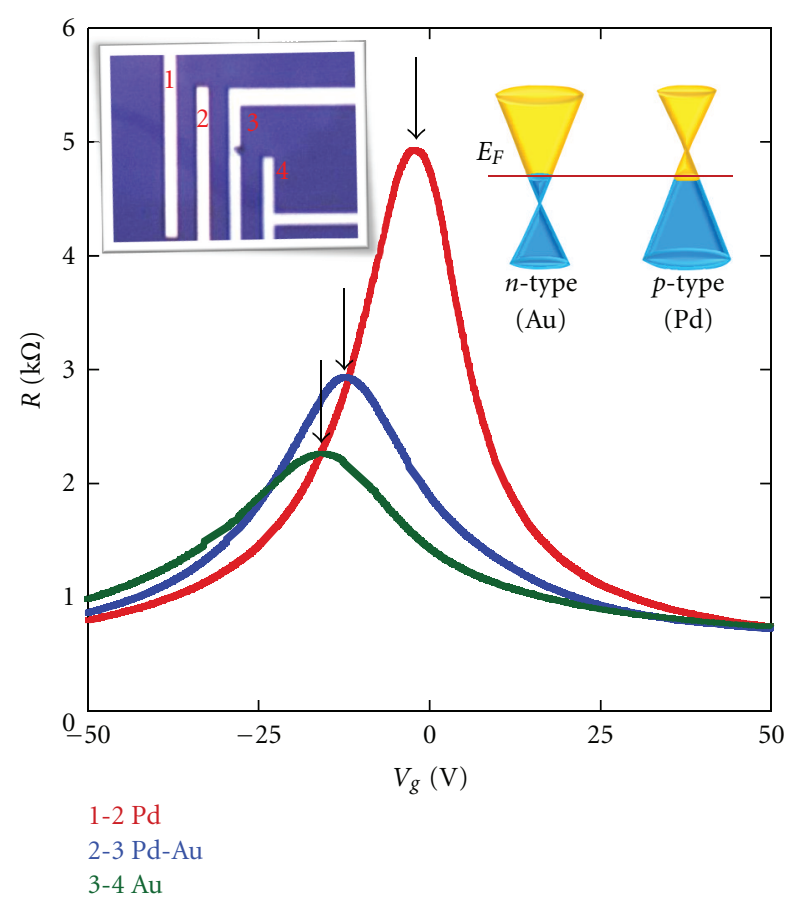

FIGURE 5: Gate response curves for a symmetric Pd-contact graphene device (1-2), an Au-contact device (3-4), and an asymmetric-contact device with $\mathrm{Pd}$ and $\mathrm{Au}$ electrodes (2-3). Inset: an optical microscope image of graphene with asymmetric heterometal electrodes ( $\mathrm{Pd}, \mathrm{Au})$.

Next, we employed asymmetric hetero-metal electrodes $(\mathrm{Au}, \mathrm{Ni})$ and $(\mathrm{Pd}, \mathrm{Au})$ in graphene devices to more clearly observe the effects of the metal work functions. Within a single device, we fabricated 4 electrodes using two different types of metal, as shown in Figures 4 and 5. Figure 4 compares the $I-V_{g}$ characteristics measured from a symmetric Au-contact graphene device, an $\mathrm{Ni}$-contact device, and an asymmetric-contact device containing $\mathrm{Ni}$ and $\mathrm{Au}$ electrodes. The measured $V_{g}^{\text {Dirac }}(\mathrm{V})$ values for the three measurement configurations were $-12.08 \mathrm{~V},-21.51 \mathrm{~V}$, and $-18.28 \mathrm{~V}$, respectively. As shown, the Dirac point of the $\mathrm{Au}-\mathrm{Ni}$-contact graphene device was situated between that of the Au-contact device and that of the Ni-contact graphene device, confirming that the work function of the contact metal indeed significantly influenced the electronic properties of graphene. A Pd electrode with a large $V_{g}^{\text {Dirac }}(\mathrm{V})$ and an Au electrode were paired to fabricate a bipolar contact device: one contact injected electrons, and the other injected holes through the respective contact barriers.

Figure 5 shows the gate response curves of the graphene device containing Pd and Au hybrid contacts. The measured $V_{g}^{\text {Dirac }}(\mathrm{V})$ values for the three measurement configurations were $-2.38 \mathrm{~V}(\mathrm{Pd}-\mathrm{Pd}),-12.27 \mathrm{~V}(\mathrm{Pd}-\mathrm{Au})$, and $-13.98 \mathrm{~V}$ $(\mathrm{Au}-\mathrm{Au})$, respectively. The $V_{g}^{\text {Dirac }}(\mathrm{V})$ of $\mathrm{Pd}-\mathrm{Pd}$ in this particular device was slightly smaller than the value $(+1.97 \mathrm{~V})$ measured for the averaged symmetric devices, as shown in Figure 2. The Pd-Au-contact graphene devices were situated between the $\mathrm{Au}$-contact device and the Pd-contact graphene

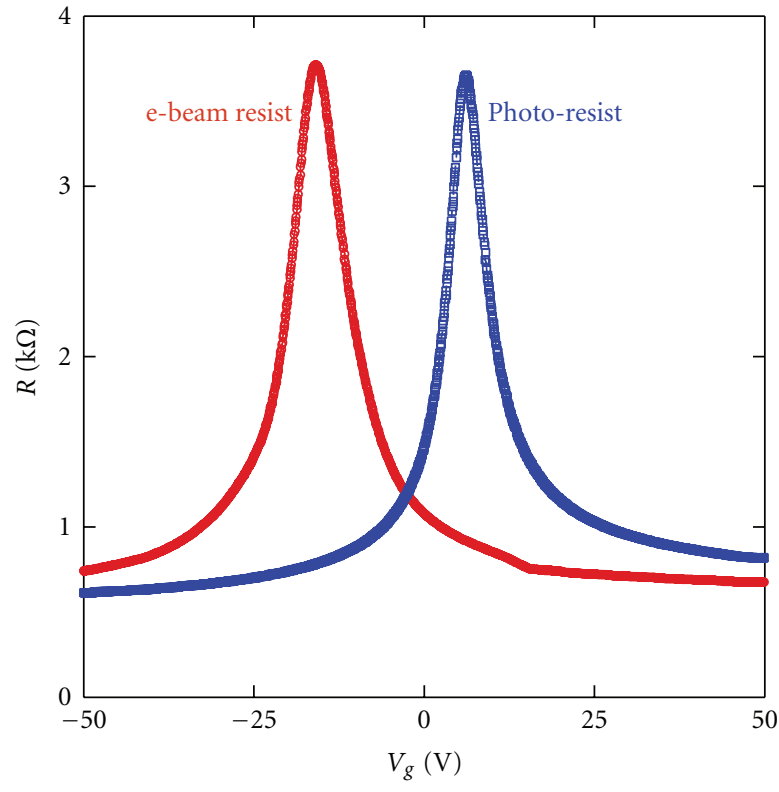

FIGURE 6: Gate response of the resistance of graphene devices produced using different resists (e-beam resist or photoresist) in the fabrication process.

device. Within the gate bias range from $-2.38 \mathrm{~V}$ to $-13.98 \mathrm{~V}$, the Fermi level for the Au contact side aligned at the electron band. On the other hand, the Fermi level for the Pd contact side was positioned at the hole band, as shown in the right inset of Figure 6. Transport measurements within this bias range may detect interesting phenomena originating from the mixed type of charge carriers in the graphene conducting channel.

Another interesting experimental observation to note is the effect of the polymeric resist on the movement of the Dirac point. When we fabricated graphene devices using a different type of resist solution (a AZ5214 photoresist instead of the PMMA electron beam resist used for the previous measurements), the resulting devices showed Dirac point voltages that were shifted by $10 \mathrm{~V}$ in the positive direction (see Figure 6). Note that the global shape of the gate response curve and the resistance did not depend on the resist used. The gate response curve shifted in a parallel direction while maintaining the curve shape commonly observed in CNT devices, which is known to arise from channel doping and not from contact modification [2]. Doping at the graphene channel surface originated from the chemical composition of the photoresist. Note that the previous experiments used the e-beam resist (PMMA) to fabricate the graphene devices. The sensitivity to the type of resist may be one reason why the $V_{g}^{\text {Dirac }}(\mathrm{V})$ appeared to be arbitrarily positioned in previous transport experiments [18-20]. Annealing processes commonly used for graphene devices may help remove the residual chemical species from the photoresist, which could minimize channel doping effects [25]. After removing the channel doping by the proper techniques, the position of the Dirac point at the interface between the metal electrode and 
the graphene should be governed by the work function of the metal-graphene complex.

\section{Summary}

We have studied the positioning of the Dirac point in graphene devices with asymmetric metal electrodes by measuring the transport properties of the devices. We found that the positioning of $V_{g}^{\text {Dirac }}(\mathrm{V})$ in hetero-metal contact devices was strongly influenced by the modified work function values of the metal-graphene complexes. The asymmetric metal contact electrodes showed Fermi levels that were aligned at one contact with the electron band and at the other contact with the hole band, within a certain bias range. This result demonstrates that it is possible to simultaneously inject different types of charge carriers into the same graphene channel. Finally, we found that the type of the resist shifted $V_{g}^{\text {Dirac }}(\mathrm{V})$ significantly via channel doping.

\section{Acknowledgments}

This paper was supported by the Chonbuk National University and the National Research Foundation of Korea (R012008-000-11425-0 and KRF-2008-313-C00319).

\section{References}

[1] Y. Nosho, Y. Ohno, S. Kishimoto, and T. Mizutani, "N-type carbon nanotube field-effect transistors fabricated by using Ca contact electrodes," Applied Physics Letters, vol. 86, no. 7, Article ID 073105, 3 pages, 2005.

[2] H.-S. Kim, B.-K. Kim, J.-J. Kim, J.-O. Lee, and N. Park, "Controllable modification of transport properties of singlewalled carbon nanotube field effect transistors with in situ $\mathrm{Al}$ decoration," Applied Physics Letters, vol. 91, no. 15, Article ID 153113, 3 pages, 2007.

[3] C. W. Lee, K. Zhang, H. Tantang et al., "Tuning of electrical characteristics in networked carbon nanotube field-effect transistors using thiolated molecules," Applied Physics Letters, vol. 91, no. 10, Article ID 103515, 2007.

[4] S. Alwarappan, G. Liu, and C.-Z. Li, "Simultaneous detection of dopamine, ascorbic acid, and uric acid at electrochemically pretreated carbon nanotube biosensors," Nanomedicine, vol. 6, no. 1 , pp. 52-57, 2010.

[5] H.-S. Kim, E.-K. Jeon, J.-J. Kim et al., "Air-stable n-type operation of Gd-contacted carbon nanotube field effect transistors," Applied Physics Letters, vol. 93, no. 12, Article ID 123106, 2008.

[6] Z. Zhang, X. Liang, S. Wang et al., "Doping-free fabrication of carbon nanotube based ballistic CMOS devices and circuits," Nano Letters, vol. 7, no. 12, pp. 3603-3607, 2007.

[7] V. Derycke, R. Martel, J. Appenzeller, and Ph. Avouris, "Controlling doping and carrier injection in carbon nanotube transistors," Applied Physics Letters, vol. 80, no. 15, p. 2773, 2002.

[8] R. Martel, T. Schmidt, H. R. Shea, T. Hertel, and Ph. Avouris, "Single- and multi-wall carbon nanotube field-effect transistors," Applied Physics Letters, vol. 73, no. 17, pp. 24472449, 1998.
[9] S. Heinze, J. Tersoff, and Ph. Avouris, "Electrostatic engineering of nanotube transistors for improved performance," Applied Physics Letters, vol. 83, no. 24, pp. 5038-5040, 2003.

[10] J. Appenzeller, J. Knoch, V. Derycke, R. Martel, S. Wind, and $\mathrm{Ph}$. Avouris, "Field-modulated carrier transport in carbon nanotube transistors," Physical Review Letters, vol. 89, no. 12, Article ID 126801, 4 pages, 2002.

[11] P. G. Collins, K. Bradley, M. Ishigami, and A. Zettl, "Extreme oxygen sensitivity of electronic properties of carbon nanotubes," Science, vol. 287, no. 5459, pp. 1801-1804, 2000.

[12] A. Bachtold, P. Hadley, T. Nakanishi, and C. Dekker, "Logic circuits with carbon nanotube transistors," Science, vol. 294, no. 5545, pp. 1317-1320, 2001.

[13] R. Martel, V. Derycke, C. Lavoie et al., "Ambipolar electrical transport in semiconducting single-wall carbon nanotubes," Physical Review Letters, vol. 87, no. 25, Article ID 256805, 4 pages, 2001.

[14] R. Saito, M. Fujita, G. Dresselhaus, and M. S. Dresselhaus, "Electronic structure of graphene tubules based on C60," Physical Review B, vol. 46, no. 3, pp. 1804-1811, 1992.

[15] F. Schedin, A. K. Geim, S. V. Morozov et al., "Detection of individual gas molecules adsorbed on graphene," Nature Materials, vol. 6, no. 9, pp. 652-655, 2007.

[16] J. B. Oostinga, H. B. Heersche, X. Liu, A. F. Morpurgo, and L. M. K. Vandersypen, "Gate-induced insulating state in bilayer graphene devices," Nature Materials, vol. 7, no. 2, pp. 151-157, 2007.

[17] I. Gierz, C. Riedl, U. Starke, C. R. Ast, and K. Kern, "Atomic hole doping of graphene," Nano Letters, vol. 8, no. 12, pp. 4603-4607, 2008.

[18] D. B. Farmer, G.-M. Roksana, V. Perebeinos et al., "Chemical doping and electron-hole conduction asymmetry in graphene devices," Nano Letters, vol. 9, no. 1, pp. 388-392, 2009.

[19] Y. Dan, Y. Lu, N. J. Kybert, Z. Luo, and A. T. C. Johnson, "Intrinsic response of graphene vapor sensors," Nano Letters, vol. 9, no. 4, pp. 1472-1475, 2009.

[20] R. Nouchi, M. Shiraishi, and Y. Suzuki, "Transfer characteristics in graphene field-effect transistors with Co contacts," Applied Physics Letters, vol. 93, no. 15, Article ID 152104, 2008.

[21] S. Alwarappan, A. Erdem, C. Liu, and C.-Z. Li, "Probing the electrochemical properties of graphene nanosheets for biosensing applications," Journal of Physical Chemistry C, vol. 113, no. 20, pp. 8853-8857, 2009.

[22] G. Giovannetti, P. A. Khomyakov, G. Brocks, V. M. Karpan, J. van den Brink, and P. J. Kelly, "Doping graphene with metal contacts," Physical Review Letters, vol. 101, no. 2, Article ID 026803, 2008.

[23] N. Park, B.-K. Kim, J.-O. Lee, and J.-J. Kim, "Influence of metal work function on the position of the Dirac point of graphene field-effect transistors," Applied Physics Letters, vol. 95, Article ID 243105, 3 pages, 2009.

[24] H. B. Michaelson, "The work function of the elements and its periodicity," Journal of Applied Physics, vol. 48, no. 11, pp. 4729-4733, 1977.

[25] K. Nagashio, T. Nishimura, K. Kita, and A. Toriumi, "Systematic investigation of the intrinsic channel properties and contact resistance of monolayer and multilayer graphene fieldeffect transistor," Japanese Journal of Applied Physics, vol. 49, no. 5, part 1, Article ID 051304, 6 pages, 2010. 

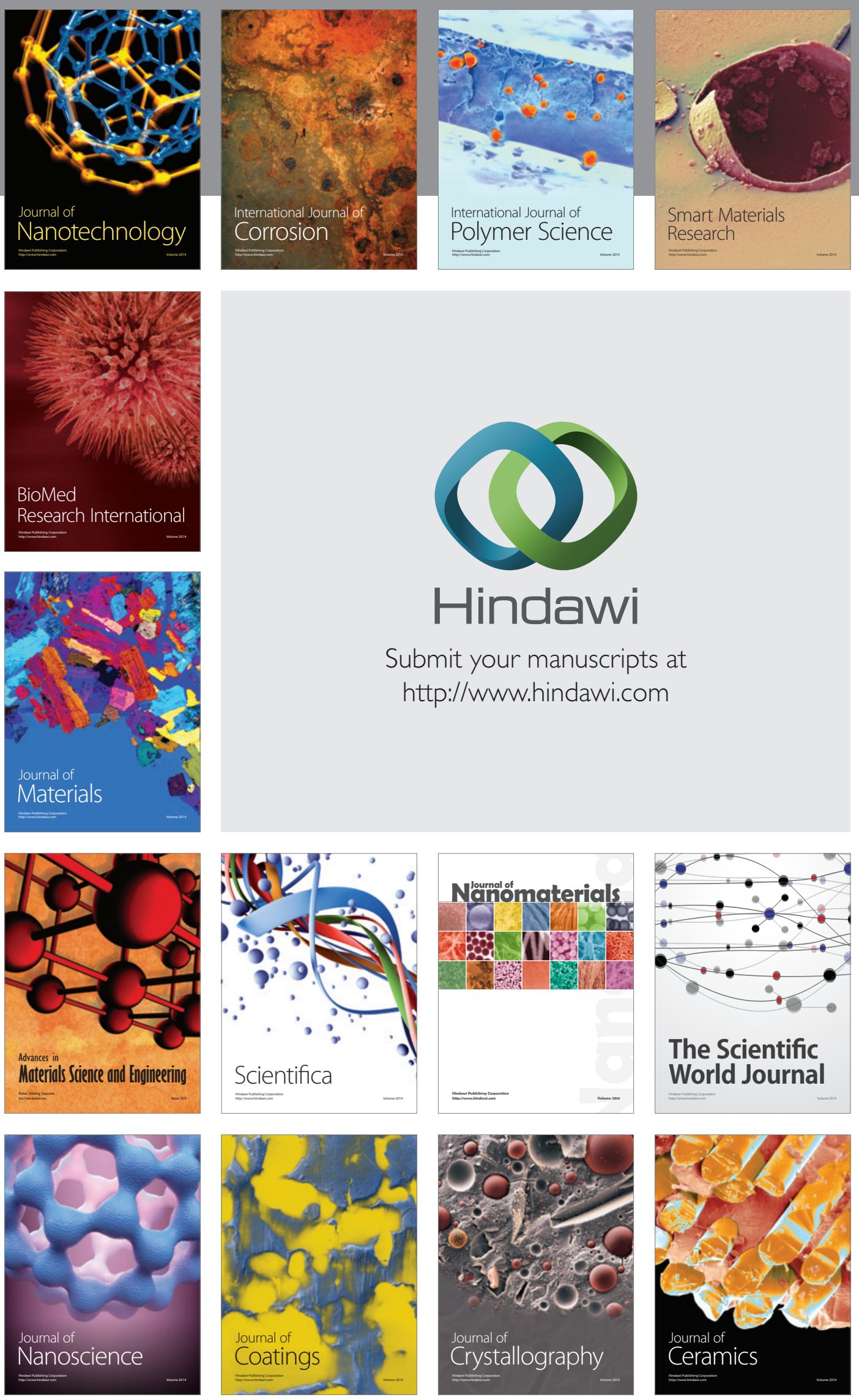

The Scientific World Journal

Submit your manuscripts at

http://www.hindawi.com

\section{World Journal}

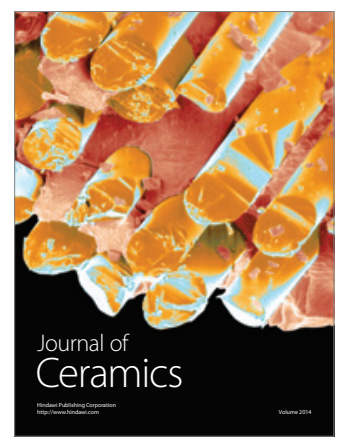

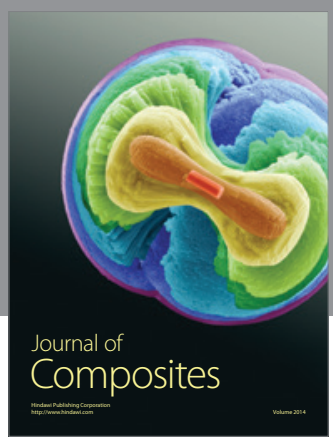
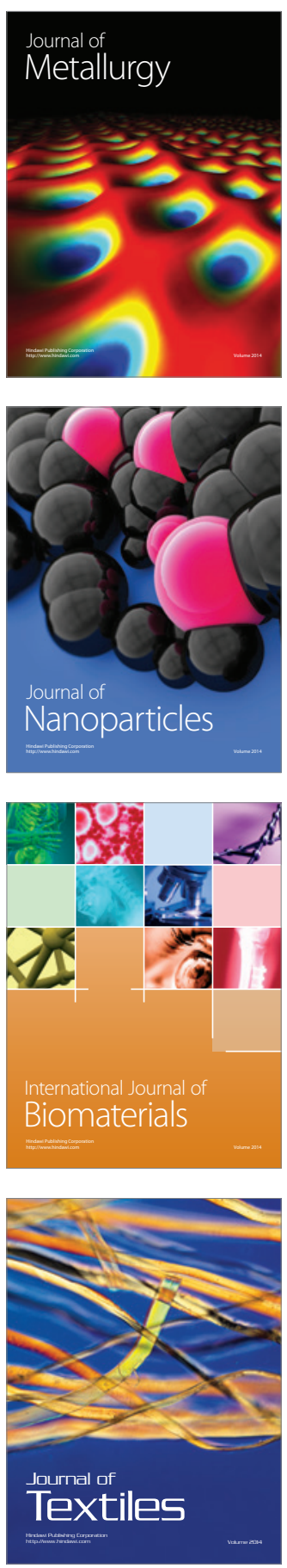